- Original Article

\title{
The Relationship between the Triglyceride to High-Density Lipoprotein Cholesterol Ratio and Metabolic Syndrome
}

\author{
Hyun-Gyu Shin, Young-Kwang Kim, Yong-Hwan Kim, Yo-Han Jung, Hee-Cheol Kang* \\ Department of Family Medicine, Yonsei University Hospital, Seoul, Korea
}

Background: Metabolic syndrome is associated with cardiovascular diseases and is characterized by insulin resistance. Recent studies suggest that the triglyceride/high-density lipoprotein cholesterol (TG/HDLC) ratio predicts insulin resistance better than individual lipid levels, including TG, total cholesterol, low-density lipoprotein cholesterol (LDLC), or HDLC. We aimed to elucidate the relationship between the TG/HDLC ratio and metabolic syndrome in the general Korean population.

Methods: We evaluated the data of adults $\geq 20$ years old who were enrolled in the Korean National Health and Nutrition Examination Survey in 2013 and 2014. Subjects with angina pectoris, myocardial infarction, stroke, or cancer were excluded. Metabolic syndrome was defined by the harmonized definition. We examined the odds ratios (ORs) of metabolic syndrome according to TG/HDLC ratio quartiles using logistic regression analysis (SAS ver. 9.4; SAS Institute Inc., Cary, NC, USA). Weighted complex sample analysis was also conducted.

Results: We found a significant association between the TG/HDLC ratio and metabolic syndrome. The cutoff value of the TG/HDLC ratio for the fourth quartile was $\geq 3.52$. After adjustment, the OR for metabolic syndrome in the fourth quartile compared with that of the first quartile was 29.65 in men and 20.60 in women $(\mathrm{P}<0.001)$.

Conclusion: The TG/HDLC ratio is significantly associated with metabolic syndrome.

Keywords: Metabolic Syndrome; Insulin Resistance; Low-Density Lipoprotein Cholesterol; Triglycerides; HighDensity Lipoprotein Cholesterol 


\section{INTRODUCTION}

Cardiovascular diseases occur more frequently in patients with metabolic syndrome. ${ }^{1)}$ In particular, in middle-aged men with metabolic syndrome the risk of total mortality due to cardiovascular diseases is 2.6-3.0 times higher than observed in individuals without metabolic syndrome. ${ }^{2)}$ Insulin resistance is important in metabolic syndrome and is associated with many diseases including coronary disease, hypertension, adult-onset diabetes, obesity, and stroke. ${ }^{3-6)}$

Dyslipidemia is also a risk factor for cardiovascular diseases. Conventionally, the role of triglycerides (TGs), high-density lipoprotein cholesterol (HDLC), and low-density lipoprotein cholesterol (LDLC) have been emphasized. High TG and low HDLC are independent risk factors of cardiovascular diseases. ${ }^{7,8)}$ TGs and HDLC are also components of metabolic syndrome. In practice, many clinicians focus primarily on LDLC in high-risk cardiovascular patients. However, $60 \%$ to $70 \%$ of cardiovascular events occur despite therapy to lower LDLC levels. ${ }^{9)}$ Also, a prior study reported that high LDLC levels do not increase the risk of mortality in women. ${ }^{10)}$

Recently, the TG/HDLC ratio was suggested as a compliment to LDLC for predicting the risk of cardiovascular diseases. The TG/HDLC ratio is significantly related to insulin resistance ${ }^{11-13)}$ and also appears to be useful in predicting the development of diabetes, ${ }^{14)}$ coronary heart disease, and cardiovascular mortality. ${ }^{15)}$ Previous studies have demonstrated that the TG/HDLC ratio is a better screening index for insulin resistance than the homeostasis model assessment for insulin resistance. $^{16)}$

It would be clinically useful to identify insulin-resistant individuals before the appearance of cardiovascular diseases. One method has been to assess the number of criteria for metabolic syndrome present in a patient. ${ }^{17)}$ Several studies have suggested that the TG/HDLC ratio can identify insulin-resistant individuals in a simpler manner. ${ }^{11-13)}$ Also, the TG/HDLC ratio has been shown to be comparable to of the use of metabolic syndrome criteria in identifying insulin resistance in apparently healthy individuals. ${ }^{18,19)}$

Data on the association between the TG/HDLC ratio and metabolic syndrome in the Korean population is limited. If the TG/HDLC ratio could be shown to be closely associated to metabolic syndrome, measurement of the TG/HDLC ratio may be clinically useful because of its simplicity. Determination of the TG/HDLC ratio does not require a measurement of waist circumference, a measurement that varies with sex and ethnicity and is not routinely checked in clinical situations. We aimed to elucidate the relationship between the TG/HDLC ratio and metabolic syndrome in the general Korean population.

\section{METHODS}

We evaluated the original data of adults aged $\geq 20$ years who were enrolled in the Korean National Health and Nutrition Examination Survey in 2013 and 2014. Subjects with a history of angina pectoris, myocardial infarction, stroke, or cancer were excluded from the study. The metabolic syndrome definition was based on the harmonized definition emphasizing variations of waist circumference according to racial groups. ${ }^{17)}$ Metabolic syndrome was defined by fulfilling $\geq 3$ components. For central obesity, the definition of the Korean Society for The Study of Obesity was used. ${ }^{20)}$ Because most drugs for the treatment of dyslipidemia (statins) primarily target the lowering of LDLC, the use of medication for dyslipidemia was ignored.

The following were the components used to assess for the presence of metabolic syndrome: (1) waist circumference $>90 \mathrm{~cm}$ (men), $>85$ cm (women); (2) hypertriglycemia TG $\geq 150 \mathrm{mg} / \mathrm{dL}$; (3) HDLC $<40 \mathrm{mg} /$ $\mathrm{dL}$ (men), <50 mg/dL (women); (4) hypertension: systolic blood pressure $\geq 130 \mathrm{~mm} \mathrm{Hg}$ or diastolic blood pressure $\geq 85 \mathrm{~mm} \mathrm{Hg}$ or on antihypertensive medication; and (5) fasting glucose $\geq 100 \mathrm{mg} / \mathrm{dL}$ or on medication for hyperglycemia.

\section{Statistical Analysis}

The original data from the 2013 and 2014 Korean National Health and Nutrition Examination were merged. Only the data of significant figures needed for analysis were used. Age (categorization), smoking status, drinking status, daytime physical activity, numbers of components of metabolic syndrome, metabolic syndrome status, quartiles for the TG/HDLC ratio, and LDLC, a chi-square test and t-test were conducted. The LDLC level was calculated using Friedewald's equation (LDLC=TC-HDLC-TG/5). The relationship between the number of metabolic syndrome components and the TG/HDLC ratio was analyzed by multiple regression, and the odds ratio (OR) of metabolic syndrome according to the TG/HDLC ratio was analyzed by logistic regression. Weighted complex sample analysis was conducted. All analyses were performed using SAS ver. 9.4 (SAS Institute Inc., Cary, NC, USA).

\section{RESULTS}

\section{General Characteristics of the Study Population}

From the total $32,875,039$ selected adults $(16,375,805$ men [ $49.81 \%$ ] and 16,499,234 women [50.19\%]), 4,200,892 men (25.65\%) and $2,956,022$ women (17.92\%) met the criteria of metabolic syndrome. In both men and women, metabolic syndrome was significantly associated with age, drinking, exercise, blood pressure, waist circumference, white blood cell count, fasting glucose, and lipid profile (Table 1). In men, the TG/HDLC ratio was $2.73 \pm 0.05$ in those without metabolic syndrome and $6.80 \pm 0.20$ in those with metabolic syndrome, a statistically significant difference $(\mathrm{P}<0.001)$. In women, the TG/HDLC ratio was $1.73 \pm 0.03$ in those without metabolic syndrome and $4.65 \pm 0.11$ in those with metabolic syndrome, also a statistically significant difference $(\mathrm{P}<0.001)$.

\section{The Relationship between the Triglyceride/High-Density Lipoprotein Cholesterol Ratio and the Number of Components of Metabolic Syndrome}

The mean value of the TG/HDLC ratio increased as the number of 


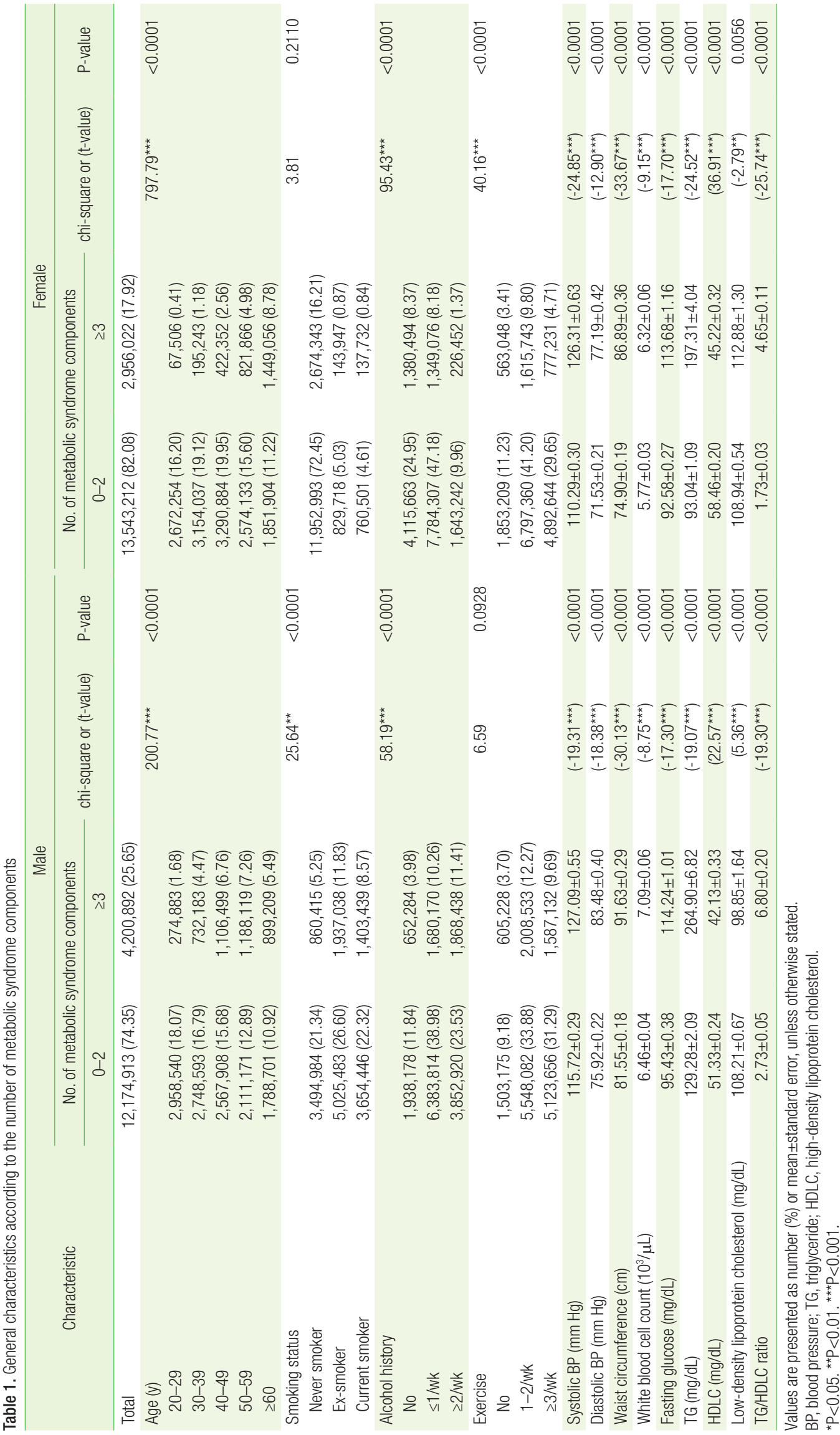


metabolic syndrome components increased: 1.61 with no components; 2.71 with 1 component; 4.20 with 2 components; 6.17 with 3 components; 7.33 with 4 components; and 10.92 with 5 components in men. In women, the mean TG/HDLC ratio was 1.17 with no components; 1.78 with 1 component; 2.92 with 2 components; 3.98 with 3 components; 5.39 with 4 components; and 6.34 with 5 components. The ratio increased significantly with the increase in the number of components present $(\mathrm{P}<0.001)$ (Table 2$)$.

\section{The Odds Ratios for Metabolic Syndrome according to the Triglyceride/High-Density Lipoprotein Cholesterol Ratio}

The TG/HDLC ratio was divided by quartile. After adjustment, the OR for metabolic syndrome in the fourth quartile compared with that of the first quartile was 29.65 in men and 20.60 in women $(\mathrm{P}<0.001)$ (Table 3).

\section{DISCUSSION}

In this study, we aimed to elucidate the relationship between the TG/ HDLC ratio and the presence of metabolic syndrome characterized by insulin resistance. The TG/HDLC ratio accordingly increased significantly in both men and women with an increase in the number of metabolic syndrome components present. In a previous study that investigated the relationship between the TG/HDLC ratio and metabolic syndrome in Koreans visiting a health-screening center, the cutoff value of the TG/HDLC ratio in the fourth quartile of was $\geq 2.93{ }^{21)}$ In pres- ent study, the fourth quartile was 3.52. The OR for metabolic syndrome was 29.65 in men and 20.60 in women.

Metabolic syndrome is characterized by insulin resistance and is primarily caused by central obesity. A recent meta-analysis showed that the total mortality rate was lower in overweight people as defined by body mass index (BMI). ${ }^{22)}$ In adult males, central obesity with normal weight defined by BMI was twice as high as the total mortality of being overweight or obese by BMI without central obesity. ${ }^{23)}$ Central obesity and insulin resistance are important components of the metabolic syndrome.

The finding that the TG/HDLC ratio is significantly related to metabolic syndrome can be explained based on multiple mechanisms. First, the TG/HDLC ratio predicts insulin resistance. ${ }^{11-13)}$ Insulin resistance promotes the accumulation of fat in the body, resulting in obesity. Insulin resistance also facilitates the progression to pre-diabetes or diabetes, and is associated with hypertension. Moreover, insulin resistance induces endothelial dysfunction of the blood vessels and is associated with oxidant stress and hormone and cytokine secretion from fat cells, resulting in inflammation that promotes the development of atherosclerosis.

Second, the TG/HDLC ratio is known to predict the level of detrimental LDLC subtypes including type B LDLC. ${ }^{24,25)}$ There are several sub-types of LDLC, and types A and B have received the greatest attention. Type A LDLC is large in volume and is not considered to be harmful. However, the smaller and denser type B LDLC is easily oxidized and triggers inflammation and atherosclerosis, resulting in arterial stiffness. ${ }^{26,27)}$ The TG/HDLC ratio is independently associated with

Table 2. TG/HDLC ratio according to the number of metabolic components present

\begin{tabular}{cccccc}
\hline \multirow{2}{*}{ No. of components } & \multicolumn{2}{c}{ Male } & & \multicolumn{2}{c}{ Female } \\
\cline { 2 - 3 } \cline { 5 - 6 } & No. (\%) & TG/HDLC ratio (95\% CL for mean) & No. (\%) & TG/HDLC ratio (95\% CL for mean) \\
\hline 0 & $4,559,816(27.85)$ & $1.61 \pm 0.03(1.56-1.66)$ & & $6,553,087(39.72)$ & $1.17 \pm 0.01(1.15-1.20)$ \\
1 & $4,044,732(24.70)$ & $2.71 \pm 0.07(2.58-2.85)$ & & $4,165,070(25.24)$ & $1.78 \pm 0.03(1.73-1.83)$ \\
2 & $3,570,365(21.80)$ & $4.20 \pm 0.13(3.94-4.45)$ & & $2,825,055(17.12)$ & $2.92 \pm 0.10(2.72-3.12)$ \\
3 & $2,701,419(16.50)$ & $6.17 \pm 0.25(5.67-6.67)$ & & $1,744,092(10.57)$ & $3.98 \pm 0.13(3.73-4.23)$ \\
4 & $1,247,920(7.62)$ & $7.33 \pm 0.34(6.66-7.99)$ & & $924,586(5.60)$ & $5.39 \pm 0.20(5.00-5.79)$ \\
5 & $251,553(1.54)$ & $10.92 \pm 1.16(8.64-13.21)$ & & $287,343(1.74)$ & $6.34 \pm 0.36(5.64-7.04)$ \\
P-trend & & $<0.001$ & & $<0.001$ \\
\hline
\end{tabular}

P-values were calculated using simple regression analysis.

TG, triglyceride; HDLC, high-density lipoprotein cholesterol; CL, confidence level.

Table 3. Odds ratios for metabolic syndrome across the TG/HDLC ratio quartiles

\begin{tabular}{|c|c|c|c|c|c|c|c|c|}
\hline \multirow{2}{*}{$\begin{array}{l}\text { Quartile of TG/ } \\
\text { HDLC ratio } \\
\text { (ratio value) }\end{array}$} & \multicolumn{4}{|c|}{ Odds ratio for male } & \multicolumn{4}{|c|}{ Odds ratio for female } \\
\hline & Unadjusted (95\% CL) & P-value & Adjusted $^{\dagger}(95 \% \mathrm{CL})$ & P-value & Unadjusted (95\% CL) & P-value & Adjusted $^{\dagger}(95 \% \mathrm{CL})$ & P-value \\
\hline Q1 $(<1.28)$ & 1 & & 1 & & 1 & & 1 & \\
\hline Q2 (1.28-2.12) & $2.76^{*}(1.23-6.19)$ & 0.0138 & $1.67(0.54-5.17)$ & 0.3734 & $1.45(0.88-2.37)$ & 0.1458 & $0.70(0.39-1.28)$ & 0.2451 \\
\hline Q3 (2.12-3.52) & $7.11^{\star \star \star}(3.39-14.92)$ & $<0.0001$ & $4.13^{\star \star}(1.44-11.87)$ & 0.0084 & $4.75^{\star \star \star}(2.80-8.06)$ & $<0.0001$ & $2.23^{\star \star}(2.02-6.16)$ & 0.0063 \\
\hline Q4 ( $\geq 3.52)$ & $28.38^{\star \star \star}(13.23-60.90)$ & $<0.0001$ & $29.65^{\star \star \star}(10.53-83.53)$ & $<0.0001$ & $16.20^{\star \star \star}(7.63-34.41)$ & $<0.0001$ & $20.60^{\star * \star}(11.33-37.44)$ & $<0.0001$ \\
\hline
\end{tabular}

TG, triglyceride; HDLC, high-density lipoprotein cholesterol; CL, confidence level.

${ }^{\star} \mathrm{P}<0.05$. ${ }^{* *} \mathrm{P}<0.01$. ${ }^{* * *} \mathrm{P}<0.001 .{ }^{\dagger}$ Adjusted for age, smoking status, alcohol history, waist circumference, HDLC, low-density lipoprotein cholesterol, blood pressure, fasting glucose, and white blood cell count. 
an increase in arterial stiffness in healthy individuals. ${ }^{28)}$

A strength of this study is that the results are reliable in terms of general applicability to the Korean population. A weakness of this crosssectional study is the lack of clinical outcomes, we cannot conclude that the TG/HDLC ratio is as powerful in predicting clinical outcomes as is the metabolic syndrome. Further studies are needed to determine which factors, including the TG/HDLC ratio, best predict the morbidity and mortality rate of cardiovascular diseases in patients with the metabolic syndrome. Because lipid profiles vary with nationality, gender, and ethnicity, additional studies are needed to determine the appropriate cutoff points of the TG/HDLC ratio among these different populations.

\section{CONFLICT OF INTEREST}

No potential conflict of interest relevant to this article was reported.

\section{REFERENCES}

1. Isomaa B, Almgren P, Tuomi T, Forsen B, Lahti K, Nissen M, et al. Cardiovascular morbidity and mortality associated with the metabolic syndrome. Diabetes Care 2001;24:683-9.

2. Lakka HM, Laaksonen DE, Lakka TA, Niskanen LK, Kumpusalo E, Tuomilehto J, et al. The metabolic syndrome and total and cardiovascular disease mortality in middle-aged men. JAMA 2002;288:2709-16.

3. Wheatcroft SB, Williams IL, Shah AM, Kearney MT. Pathophysiological implications of insulin resistance on vascular endothelial function. Diabet Med 2003;20:255-68.

4. DeFronzo RA, Ferrannini E. Insulin resistance: a multifaceted syndrome responsible for NIDDM, obesity, hypertension, dyslipidemia, and atherosclerotic cardiovascular disease. Diabetes Care 1991;14:17394.

5. Kahn BB, Flier JS. Obesity and insulin resistance. J Clin Invest 2000;106:473-81.

6. Kahn SE, Hull RL, Utzschneider KM. Mechanisms linking obesity to insulin resistance and type 2 diabetes. Nature 2006;444:840-6.

7. Egger M, Smith GD, Pfluger D, Altpeter E, Elwood PC. Triglyceride as a risk factor for ischaemic heart disease in British men: effect of adjusting for measurement error. Atherosclerosis 1999;143:275-84.

8. Sharrett AR, Ballantyne CM, Coady SA, Heiss G, Sorlie PD, Catellier D, et al. Coronary heart disease prediction from lipoprotein cholesterol levels, triglycerides, lipoprotein(a), apolipoproteins A-I and B, and HDL density subfractions: the Atherosclerosis Risk in Communities (ARIC) Study. Circulation 2001;104:1108-13.

9. Shah PK, Kaul S, Nilsson J, Cercek B. Exploiting the vascular protective effects of high-density lipoprotein and its apolipoproteins: an idea whose time for testing is coming, part I. Circulation 2001;104:2376-83.

10. Ogushi Y, Hamazaki T, Kirihara Y. Blood cholesterol as a good marker of health in Japan. World Rev Nutr Diet 2009;100:63-70.

11. McLaughlin T, Abbasi F, Cheal K, Chu J, Lamendola C, Reaven G. Use of metabolic markers to identify overweight individuals who are insulin resistant. Ann Intern Med 2003;139:802-9.

12. McLaughlin T, Reaven G, Abbasi F, Lamendola C, Saad M, Waters D, et al. Is there a simple way to identify insulin-resistant individuals at in- creased risk of cardiovascular disease? Am J Cardiol 2005;96:399-404.

13. Kim JS, Kang HT, Shim JY, Lee HR. The association between the triglyceride to high-density lipoprotein cholesterol ratio with insulin resistance (HOMA-IR) in the general Korean population: based on the National Health and Nutrition Examination Survey in 2007-2009. Diabetes Res Clin Pract 2012;97:132-8.

14. De Leon AC, Coello SD, Gonzalez DA, Diaz BB, Rodriguez JC, Hernandez AG, et al. Impaired fasting glucose, ancestry and waist-to-height ratio: main predictors of incident diagnosed diabetes in the Canary Islands. Diabet Med 2012;29:399-403.

15. Hadaegh F, Khalili D, Ghasemi A, Tohidi M, Sheikholeslami F, Azizi F. Triglyceride/HDL-cholesterol ratio is an independent predictor for coronary heart disease in a population of Iranian men. Nutr Metab Cardiovasc Dis 2009;19:401-8.

16. Liang J, Fu J, Jiang Y, Dong G, Wang X, Wu W. TriGlycerides and highdensity lipoprotein cholesterol ratio compared with homeostasis model assessment insulin resistance indexes in screening for metabolic syndrome in the Chinese obese children: a cross section study. BMC Pediatr 2015;15:138.

17. Alberti KG, Eckel RH, Grundy SM, Zimmet PZ, Cleeman JI, Donato KA, et al. Harmonizing the metabolic syndrome: a joint interim statement of the International Diabetes Federation Task Force on Epidemiology and Prevention; National Heart, Lung, and Blood Institute; American Heart Association; World Heart Federation; International Atherosclerosis Society; and International Association for the Study of Obesity. Circulation 2009;120:1640-5.

18. Salazar MR, Carbajal HA, Espeche WG, Leiva Sisnieguez CE, March $\mathrm{CE}$, Balbin E, et al. Comparison of the abilities of the plasma triglyceride/high-density lipoprotein cholesterol ratio and the metabolic syndrome to identify insulin resistance. Diab Vasc Dis Res 2013;10:346-52.

19. Sung KC, Reaven G, Kim S. Ability of the plasma concentration ratio of triglyceride/high-density lipoprotein cholesterol to identify increased cardio-metabolic risk in an East Asian population. Diabetes Res Clin Pract 2014;105:96-101.

20. Kim MK, Lee WY, Kang JH, Kang JH, Kim BT, Kim SM, et al. 2014 Clinical practice guidelines for overweight and obesity in Korea. Korean J Obes 2014;23:217-21.

21. Oh SY, Kim SH, Hwang IC. Relationship between metabolic syndrome and triglyceride/high-density lipoprotein cholesterol ratio. Korean J Clin Geri 2014;15:69-74.

22. Flegal KM, Kit BK, Orpana H, Graubard BI. Association of all-cause mortality with overweight and obesity using standard body mass index categories: a systematic review and meta-analysis. JAMA 2013;309:71-82.

23. Sahakyan KR, Somers VK, Rodriguez-Escudero JP, Hodge DO, Carter RE, Sochor O, et al. Normal-weight central obesity: implications for total and cardiovascular mortality. Ann Intern Med 2015;163:827-35.

24. Dobiasova M, Frohlich J. The plasma parameter log (TG/HDL-C) as an atherogenic index: correlation with lipoprotein particle size and esterification rate in apoB-lipoprotein-depleted plasma (FERHDL). Clin Biochem 2001;34:583-8.

25. Hanak V, Munoz J, Teague J, Stanley A Jr, Bittner V. Accuracy of the triglyceride to high-density lipoprotein cholesterol ratio for prediction of the low-density lipoprotein phenotype B. Am J Cardiol 2004;94:21922. 
26. Austin MA, Breslow JL, Hennekens CH, Buring JE, Willett WC, Krauss RM. Low-density lipoprotein subclass patterns and risk of myocardial infarction. JAMA 1988;260:1917-21.

27. Crouse JR, Parks JS, Schey HM, Kahl FR. Studies of low density lipoprotein molecular weight in human beings with coronary artery dis- ease. J Lipid Res 1985;26:566-74.

28. Wen JH, Zhong YY, Wen ZG, Kuang CQ, Liao JR, Chen LH, et al. Triglyceride to HDL-C ratio and increased arterial stiffness in apparently healthy individuals. Int J Clin Exp Med 2015;8:4342-8. 